\title{
Research Article \\ Collaborative Image Coding and Transmission over Wireless Sensor Networks
}

\author{
Min $\mathrm{Wu}^{1}$ and Chang Wen Chen ${ }^{2}$ \\ ${ }^{1}$ MAKO Surgical Corporation, Fort Lauderdale, FL 33317, USA \\ ${ }^{2}$ Department of Electrical and Computer Engineering, Florida Institute of Technology (FIT), Melbourne FL32901, USA
}

Received 6 February 2006; Revised 3 August 2006; Accepted 13 August 2006

Recommended by Chun-Shien Lu

The imaging sensors are able to provide intuitive visual information for quick recognition and decision. However, imaging sensors usually generate vast amount of data. Therefore, processing and coding of image data collected in a sensor network for the purpose of energy efficient transmission poses a significant technical challenge. In particular, multiple sensors may be collecting similar visual information simultaneously. We propose in this paper a novel collaborative image coding and transmission scheme to minimize the energy for data transmission. First, we apply a shape matching method to coarsely register images to find out maximal overlap to exploit the spatial correlation between images acquired from neighboring sensors. For a given image sequence, we transmit background image only once. A lightweight and efficient background subtraction method is employed to detect targets. Only the regions of target and their spatial locations are transmitted to the monitoring center. The whole image can then be reconstructed by fusing the background and the target images as well as their spatial locations. Experimental results show that the energy for image transmission can indeed be greatly reduced with collaborative image coding and transmission.

Copyright ( ) 2007 M. Wu and C. W. Chen. This is an open access article distributed under the Creative Commons Attribution License, which permits unrestricted use, distribution, and reproduction in any medium, provided the original work is properly cited.

\section{INTRODUCTION}

Networked microsensor technology is becoming one of the key technologies for the 21st century. Such sensor networks are often designed to perform tasks such as detecting, classifying, localizing, and tracking of one or more targets in the sensor fields $[1,2]$. Among all types of sensors, the imaging sensors are able to provide intuitive visual information for quick recognition and decision. However, imaging sensors usually generate vast amount of image data. Therefore, for battery-powered sensors, the transmission of image data collected in a sensor network presents the most challenging problem.

A number of research efforts are currently under way to address the issues on collaborative signal and information processing in distributed microsensor networks [3-6]. Pradhan et al. proposed a distributed coding framework to realize the coding gain of correlated data from Slepian-Wolf coding theorem in information theory [3]. Ideally, no information needs to be exchanged among correlated sensors during the encoding process. At the decoder, data can be recovered by reaping the full benefit of the correlation between neighbor- ing sensor data. A very simple example is given to demonstrate the feasibility of this coding framework. Many researches are moving forward to distributed image and video coding based on Wyner-Ziv theorem which is an extension to lossy coding from Slepian-Wolf theorem [7-9]. Pradhan proposed a syndrome-based multimedia coding [10]. Girod presented distributed video coding using turbo code [8]. However, the quality of reconstructed video is limited by the accuracy of the prediction as side information from motion estimation at the decoding side. Girod also applied Wyner-Ziv coding to distributed image compression for large camera arrays [9]. To acquire a good estimate for the Wyner-Ziv coded views, conventional cameras have to be interspersed among sensor nodes.

Wagner et al. proposed another distributed image compression scheme for sensor network [6] that is different from the Slepian-Wolf and Wyner-Ziv coding. They use image matching method to register correlated views to identify maximal overlap, and send the low-resolution overlapped areas to the receiver. At the receiver, super-resolution recovery techniques are applied to reconstruct a highresolution version of the overlapped areas. In their work, 
they only exploit spatial redundancy among the images. The super-resolution overlap recovery requires multiple frames of low-resolution overlap. Thus the imaging sensors need to be deployed very densely to accommodate such technique.

In this paper, we propose to build a collaborative image coding and transmission system over distributed wireless sensor network. We consider exploiting both spatial and temporal correlations among the sensor images to reduce overall energy consumption on data transmission and processing. In our system, we assume that image sensors transmit collected image data to the monitoring center via multiple hops. Sensor nodes on the route to the monitoring center could access image data collected from previous hops. This assumption conforms to many energyefficient MAC protocols, such as data centric, hierarchical, and location-based protocols [11]. Since sensor node has very limited processing power, image processing algorithm perferably will be lightweighted and efficient and suitable for these practical applications. To exploit the spatial correlation between neighboring sensor images, a shape matching method [12] is applied to find out maximal overlapped areas. The shape matching algorithm is operated on a very small number of the feature points, hence the computational complexity can be greatly reduced. A transformation is then generated according to the matching result. We code the original image and the difference between the reference image and the transformed image. Then we transmit the coded bit stream together with the transformation parameters.

In our intended application as surveillance, we assume that the imaging sensors and their background scenes remain stationary over the entire image acquisition process. To exploit the temporal correlation among images in the same sensor, we transfer background image only once during any triggered event and transmit images when one or more targets are detected. A simple background subtraction method that is robust to global illumination change is applied to detect targets. Whenever targets are detected, only the regions of targets and their spatial locations are transmitted to the monitoring center. At the monitoring center, the whole image can be reconstructed by fusing the background and the target areas.

Since it has been proven that the power consumption for data processing is much less than that for data communication, we expect that energy saving from reduced data communication will significantly outweight the additional energy consumption from additional image matching and processing. Experimental results show that the energy for image transmission can indeed be greatly reduced with collaborative image coding.

The rest of this paper is organized as follows. In Section 2, we describe in detail our approach to the proposed collaborative image coding and transmission over distributed wireless sensor networks. Experimental results are presented in Section 3 to confirm the energy efficiency of the proposed approach. Section 4 concludes this paper with a summary and some discussions.

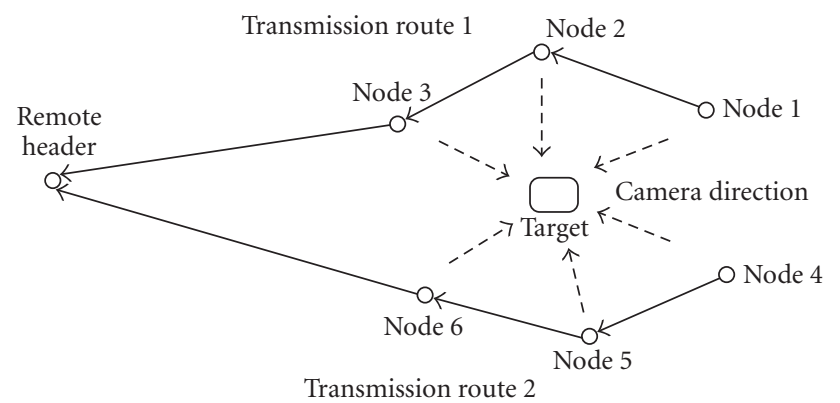

FIGURE 1: Diagram of sensor network.

\section{THE PROPOSED APPROACH}

\subsection{Exploiting spatial correlation via image matching}

In this work, we address the problem that imaging sensors are relatively densely deployed for surveillance as shown in Figure 1. Images in neighboring sensors are assumed spatially correlated with typical overlaps as shown by the top images in Figure 5. Transmitting the whole images independently means that the image data received by the monitoring center will have significant redundancy among images collected from neighboring sensors. Data transmission in this fashion will significantly shorten the sensor's life time due to unnecessary waste in the limited transmission power. This makes local on-board data compression a more energy efficient choice in low bandwidth lossy sensor networks [13]. We can reduce the spatial redundancy between the neighboring sensors so as to minimize the energy for transmission. Notice that we assume that sensor nodes communication is a multi-hop fashion from sensor nodes to the monitoring center. After one sensor sends its image to its neighboring sensor along the route to the monitoring center, an image matching method [12] can be applied to find out the maximal overlap between the image acquired by the current sensor and the image received from the previous hop. We adopt a computationally lightweight scheme to exploit the spatial correlation between two neighboring images. This technique allows for effective description of similar images in terms of only their critical feature points via a shape descriptor known as shape context. The shape context is a description of the coarse distribution of the gray scale in a neighboring area centered on a given feature point. As this method uses a small set of image feature points, it is preferrable for imaging sensors with limited battery resource and computational capability.

The proposed image matching scheme is robust and suitable for implementation in a energy constrained sensor network. When an image is sent to a neighboring sensor, the neighboring sensor computes registrations between the transmitted image and the image taken by the neighboring sensor. The transmitted image is referred to as original image; the image taken by the neighboring sensor is referred to as reference image. For simplicity, the dominant edges on both images are extracted from the downsampled images. Any standard edge detection algorithm, such as Sobel 


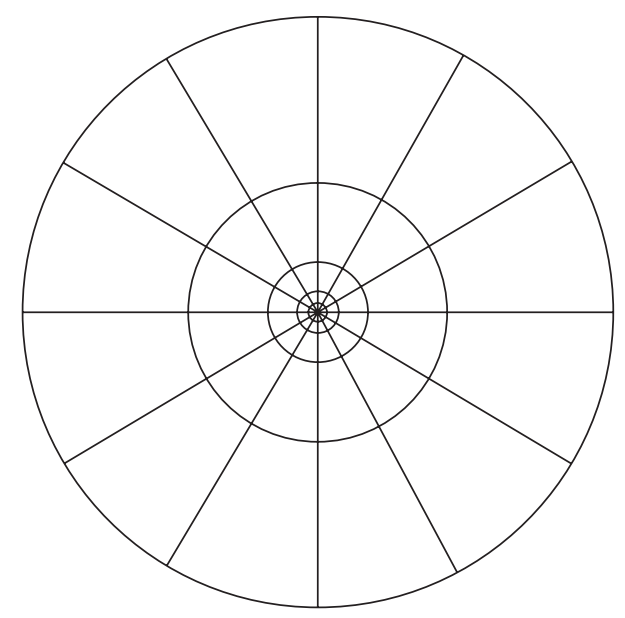

(a)

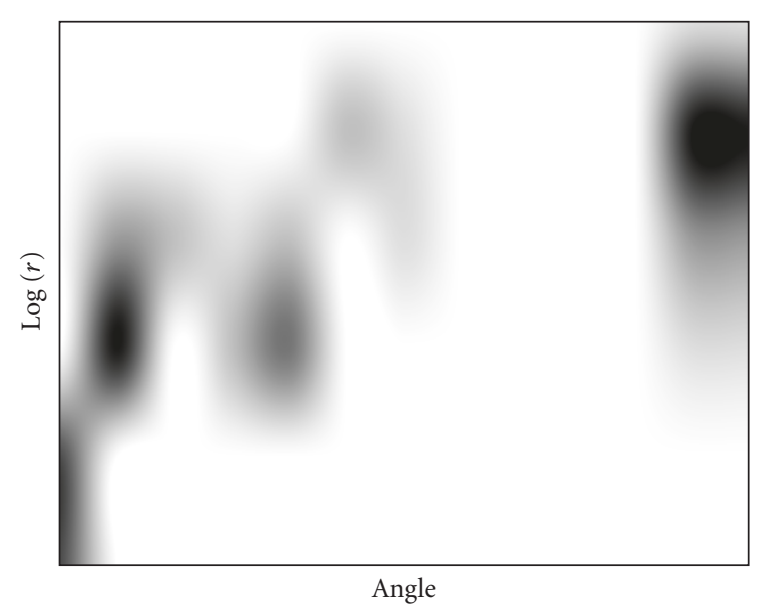

(b)

FIGURE 2: log-polar histogram bins and a shape context for one feature point.

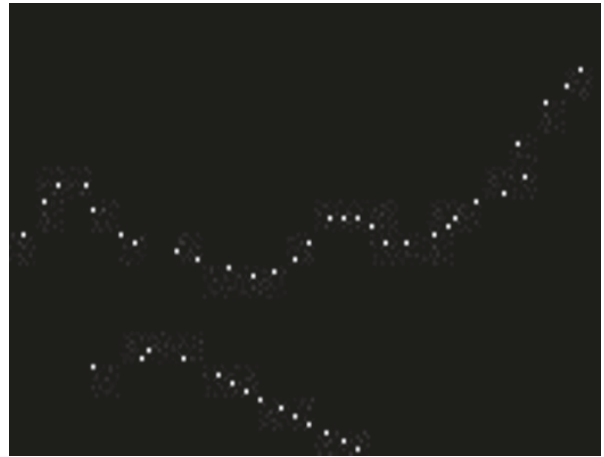

(a)

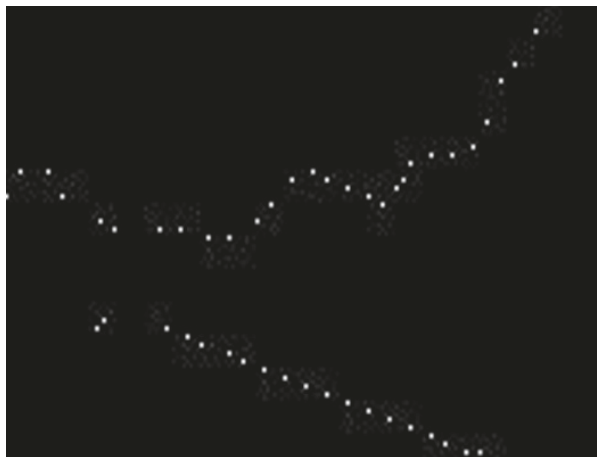

(b)

FIgURE 3: Feature point sets extracted from two neigboring images.

operator, can be employed in this step. In the detection of dominant edges, a threshold for edge detection algorithm is selected so that only a presetted number of edge points are detected for this threshold. The feature points are then extracted from the edge points such that the feature points are evenly spaced along the edges. Then, in both feature point sets, for each point a shape context is computed. The shape context is a coarse histogram description operated on feature point set [12]. The histogram is determined by the number of feature points located in the bins shown in Figure 2(a). For a feature point $p_{i}$ on the shape, the histogram $h_{i}$ is calculated as

$$
h_{i}(k)=\#\left\{q \neq p_{i}:\left(q-p_{i}\right) \in \operatorname{bin}(k)\right\},
$$

where $q$ is feature point and $k$ is the index of bins. The bins are centered on the feature point and uniform in log-polar space, making the descriptor more sensitive to positions of nearby points than farther away points. Figure 2(b) shows a shape context on one feature point in Figure 3. After two sets of shape contexts are extracted from two correlated images, a bipartite graph matching is employed to find the best one-toone match between two sets of points. The cost of matching two points on two shapes is defined as

$$
C_{i, j}=C\left(p_{i}, q_{j}\right)=\frac{1}{2} \sum_{k=1}^{K} \frac{\left[h_{i}(k)-h_{j}(k)\right]^{2}}{h_{i}(k)+h_{j}(k)},
$$

where $p_{i}$ is a feature point in one image, and $q_{j}$ is a feature point in the other image. We minimize the total cost of matching to find the best one-to-one match:

$$
H=\sum_{i, j} C\left(p_{i}, q_{j}\right) .
$$

Once the correspondence of two shapes is obtained. The correspondence of arbitrary pixels on two images is defined as a plane transform that is defined as

$$
f(x, y)=a_{1}+a_{x} x+a_{y} y .
$$


From the finite set of correspondences between points on two shapes, the coefficients of $a$ are estimated by least mean square solution:

$$
P a=v,
$$

where $f\left(x_{i}, y_{i}\right)=v_{i}$ is corresponding locations when $p_{i}=$ $\left(x_{i}, y_{i}\right) . a$ is a vector $\left(a_{1}, a_{x}, a_{y}\right) . P$ is a matrix of coordinates of the feature points:

$$
P=\left(\begin{array}{ccc}
1 & x_{1} & y_{1} \\
: & : & : \\
1 & x_{n} & y_{n}
\end{array}\right)
$$

The registrations allow us to identify the largest region of overlap as described above. Once we get the coefficients $a$ through (5), we use two separate functions shown in (7) to model a coordinate transform to generate a warped image,

$$
T(x, y)=\left(f_{x}(x, y), f_{y}(x, y)\right) .
$$

The warped image has the best match with the reference image, and this means that the two images have the maximal overlap. We code the original image and the difference between the reference image and the warped image. Then we transmit the coded bit stream together with the transformation parameters $a$ to the next neighboring senor along the route to the monitoring center. This will reduce the energy on communication compared with transmitting two images independently.

\subsection{Exploiting temporal correlation via background subtraction}

In our research, we assume that the sensor network is intended for surveillance. An event driven strategy can be adopted for energy efficient deployment $[14,15]$. In this case, the sensors can be put into "sleep" state if no target has been detected via nonimaging sensor $[15,16]$. Once a target is detected by a nonimaging sensor, the imaging sensors will wake up to work and the imaging sensor-based target tracking stage will begin. We assume that the imaging sensors and background scene remain relatively stationary during the tracking stage. To further save energy consumption, scene change detection can be implemented such that if the scene does not change, sensor should not transmit image to the monitoring center. When one or more targets are detected, the imaging sensor will locate the target areas on the image and transmit only the target areas together with their spatial locations to the monitoring center. This will further reduce the energy consumption on communication at the cost of increasing signal processing energy on target detection. At the monitoring center, the image is reconstructed by fusing the background and the target areas.

We adopt background subtraction method to detect target. This is a lightweight and efficient way for target detection. A number of background subtraction methods have been proposed in recent years [17-20]. The basic idea of background subtraction algorithm can be briefly described as computing the color or intensities of pixels in the incoming image and reference image. Significant differences in the intensities from reference image were attributed to the targets. The intensity-based subtraction is sensitive to the illumination changes, because illumination changes increase the deviation of the background pixels from the original captured background images. Mittal and Huttenlocher proposed a model to represent pixels in the scene [18]. They constructed a background model to detect moving objects in video sequences. Javed et al. proposed a hierarchical approach for robust background subtraction [17]. They also used a statistical model to classify pixels whether belonging to foreground or background.

As the imaging sensor has limited signal processing power, lightweight and efficient target detection is desirable in the application. To deal with the illumination changes, we could update background at a short time interval to keep the illumination changes under a fixed threshold. However, this will increase the burden of image transmission. Another solution is employing background subtraction in gradient image. The basic idea is that any foreground region that corresponds to an actual object will have high values of gradientbased background difference at its boundaries; any slow illumination changes could be eliminated in gradient image. The gradients are calculated from the gray level image. Let $I$ be the current image and $\Delta$ be the gradient feature vector of its gray levels. We use $\Delta=\left|\Delta_{m}, \Delta_{d}\right|$ as a feature vector for gradient-based background differencing, where $\Delta_{m}$ is the gradient magnitude, that is, $\sqrt{d_{x}^{2}+d_{y}^{2}}$ and $\Delta_{d}$ is the gradient direction, that is, $\tan ^{-1}\left(d_{y} / d_{x}\right)$. For any region $R_{a}$ that corresponds to some foreground objects in the scene, there will be a high gradient at $\partial R_{a}$ on the image $I$, where $\partial R_{a}$ is the set of boundary pixels $(i, j)$ of region $R_{a}$. Thus it is reasonable to assume that $\Delta$ will have high deviation from the gradient background model at the boundary pixels. For each newly captured image, gradient magnitude and the direction values are computed. If for a certain gradient vector, the difference from the background gradient vector is greater than a preselected threshold, the pixel belongs to foreground, otherwise, it belongs to background.

We should point out that there are two types of errors in the target detection step. The first type is missing target. In this case, there is a target in the image, but the system is unable to detect it. The second type is erroneous target detection. In this case, the system detects a "target" that is not a true target. In background subtraction, most detection errors are the second type of errors. When such a detection error occurs in the process, the sensor transmits a freak target to the monitoring center. At the monitoring center, this type of error will not influence the monitoring and surveillance task since it can be easily recognized as a detection error. The freak target may be due to the variation of background scene or abrupt illumination change.

\subsection{Collaborative image coding}

In this system, we assume that each sensor has a processor to acquire images and perform background subtraction, and 
feature-based image matching. Both spatial and temporal correlations have been exploited, and three types of images are generated: whole original image, difference image, and small scale target area image. The goal of the collaborative image coding is to reduce the transmission power consumption of this imaging sensor network.

Images are distributedly compressed in an efficient and timely manner. There are many choices to compress all three types of images. The state-of-the-art coding methods include SPIHT, JPEG2000, and H.264 intra-mode. Since wireless channels are highly error pone in sensor network, and sensor images are captured in very low frequency, fully scalable image coding is very desirable in the sensor network application. H.264 intra-mode has high coding efficient and low complexity by using integer transform and intra-prediction mode. However, it does not provide progressive coding that is desirable for error prone channel in sensor network. SPIHT provides high coding efficiency in a fully progressive fashion: images can be reconstructed with any length of received encoded bit stream. We use SPIHT algorithm to compress all three types of images: whole image, difference image, and small scale target area image.

At the monitoring center, the original image and the difference between the reference image and the warped image are first decoded. Transforming original image using transformation parameters generates the warped image. The warped image plus the difference from the reference image will generate the image from the neighboring sensor. The reconstructed target image will fuse with the background image to generate the image for the purpose of surveillance.

\subsection{Collaborative image transmission}

Consider the sensor network shown in Figure 1, the goal for collaborative image transmission is to reduce the transmission energy, or equivalently, reducing the total data amount, while maintaining adequate quality of the reconstruction from all image sensors within the cluster. At the beginning, each sensor transmits its background scene only once to the monitoring center. The gradient vectors are also computed on background image and saved as the reference. Each sensor takes pictures at a fixed interval. The background subtraction method described above is employed on each captured image. Whenever one or more targets are detected, the target areas and their spatial locations are transmitted to the monitoring center. At the monitoring center, the receiver is able to reconstruct the whole image by fusing the background data with target image as well as its spatial locations information. The procedure of collaborative image transmission in Figure 1 can be summarized as follows.

(1) Transmission operations.

(a) Transmit the background of the target along the route of sensor 1 , sensor 2 , sensor 3 , and remote sensor and another route of sensor 4 , sensor 5 , sensor 6, and remote sensor, respectively.

(b) At sensors 2, 3, 5, and 6 apply the algorithm in Section 2.1 to remove spatial redundancy be-

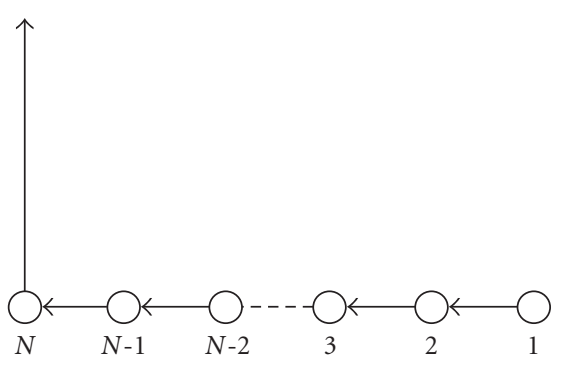

(a)

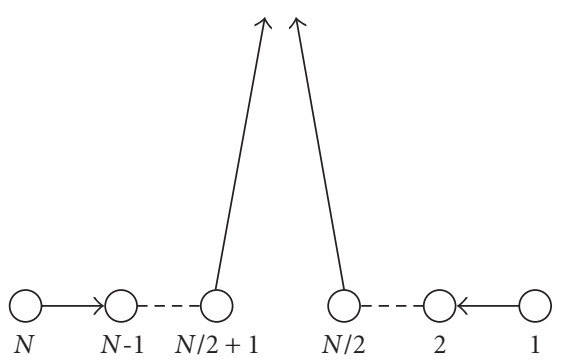

(b)

FIgURE 4: Two routing schemes.

tween images in sensors 1 and 2, sensors 2 and 3, sensors 4 and 5 , and sensors 5 and 6, respectively.

(c) At each sensor, whenever a target is detected by applying the algorithm in Section 2.2 on a new captured image, the extracted target area and its spatial location are transmitted to the remote sensor along the same route.

(2) Reconstruction operations at the monitoring center.

(a) Restore the background image transmitted from each sensor.

(b) Reconstruct sensor images by fusing background and target area as well as its spatial location each time after target image and its spatial location are received.

In summary, only one full background image needs to be transmitted from each sensor. Whenever targets are detected, only target area and its spatial location need to be transmitted to the monitoring center. At the monitoring center, the whole image can be reconstructed by the fusion of the background data and the target as well as its spatial location.

Since a distributed sensor network has multiple paths from the source to the destination, different routings may result in different network performance, such as delay and network life. Figure 4 shows two simple routing schemes. Suppose that in both schemes each sensor captures one image and transmits to the monitoring center. We denote $i$ th original image with $I_{i}$, the difference between image $i$ and the wrapped image $i-1$ with $D_{i, i-1}$, image matching between image $i$ and image $i-1$ with $M_{i, i-1}$. In Figure 4(a), 


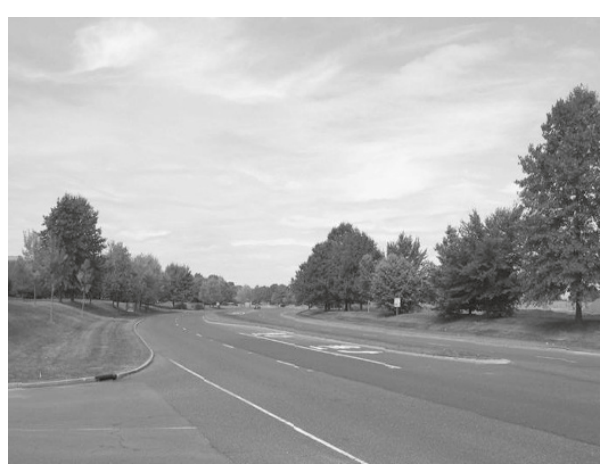

(a)

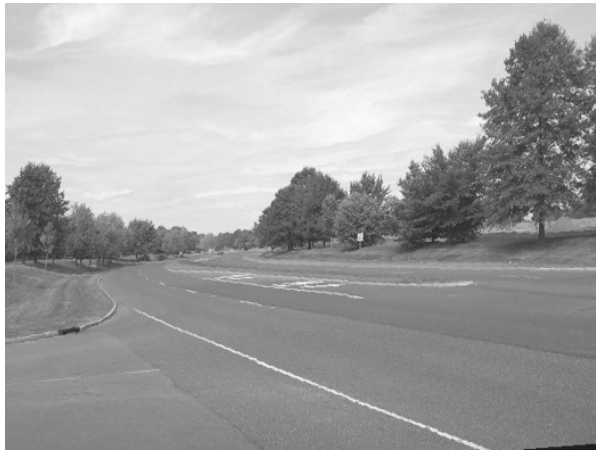

(b)

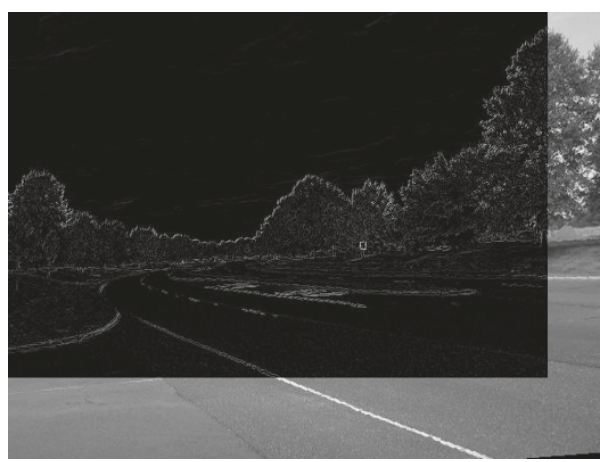

(c)

FIGURE 5: Two neighboring images and their warping difference.

sensor 1 encodes $I_{1}$, and transmits to sensor 2 , sensor 2 decodes $I_{1}$, performs image matching $M_{2,1}$, and encodes $D_{2,1}$. Sensor $N$ decodes $I_{1}, D_{2,1}, D_{3,2}, \ldots, D_{N-1, N-2}$, performs image matching $M_{N, N-1}$, encodes $D_{N, N-1}$, and transmits $I_{1}, D_{2,1}, D_{3,2}, \ldots, D_{N, N-1}$. Figure 4 (b) shows another scheme, image 1 and $\mathrm{N}$ reach monitoring center via $N / 2$ hops. For simplicity, we ignore the subscripts in calculating the total number of operations in image processing and transmission. With collaborative image processing, in total Figure 4(a) encodes $I+(N-1) \times D$, decodes $(N-1) \times I+(N-1)(N-2) / 2 \times D$, performs $(N-1) \times M$, and transmits $N \times I+N(N-1) / 2 \times D$. Figure $4(\mathrm{~b})$ encodes $2 \times I+(N-2) \times D$, decodes $(N-2) \times I+$ $(N-2)(N-4) / 4 \times D$, performs $(N-2) \times M$, and transmits $N \times I+(N+1)(N-1) / 4 \times D$.

Without collaborative image processing, Figure 4(a) encodes $N \times I$ and transmits $(N+1) N / 2 \times I$; Figure $4(\mathrm{~b})$ encodes $N \times I$ and transmits $(N+2) N / 4 \times I$. The evaluation of energy consumption will be addressed in next section. With collaborative image processing, apparently, Figure 4(b) has less image operations and fewer bits in transmission. Also in Figure 4(a) sensor $N$ consumes much more energy than that of sensor 1, this unbalance in energy drain will reduce the overall network lifetime. This analysis helps to choose the topology of sensor network and routing. The total number of hops will be as small as possible.

\section{EXPERIMENTAL RESULTS}

The experiment is conducted on the imaging sensors deployed as shown in Figure 1. The average distance between the neighboring sensors is 10 meters. The size of each image taken by imaging sensor is $384 \times 288$. Intel StrongARM SA 1110 and National Semiconductor LMX 3162 are used as processor and transceiver, respectively, in sensor node. LMX 3162 works in $2.4 \mathrm{GHz}$ unlicensed band. The transmission power is $80 \mathrm{~mJ}$ when sending data. The transmission rate is $1 \mathrm{Mbps}$. We only consider the application layer in sensor communication. Two sensor transmission routes are shown in Figure 1. One route is from sensor 1, sensor 2, sensor 3, to the remote sensor. The other route is from sensor 4 , sensor 5 , sensor 6, to the remote sensor. Each sensor is deployed to monitor traffic condition on a road.

\subsection{Image matching to exploit spatial correlation}

Two views of a scene taken from different sensors are shown as in the top two images in Figure 5. Forty five feature points are extracted from the dominant edges on two images. Figure 3 shows feature points extracted from the top two images in Figure 5. Figure 5(a) is used as original image, and Figure 5(b) is used as reference image. After bipartite graph 


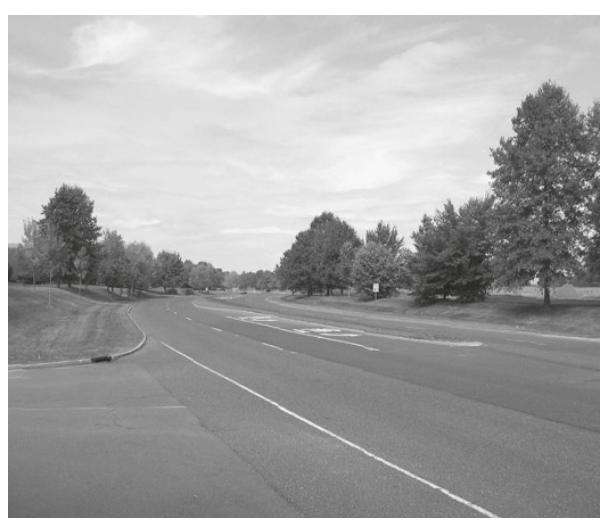

(a)

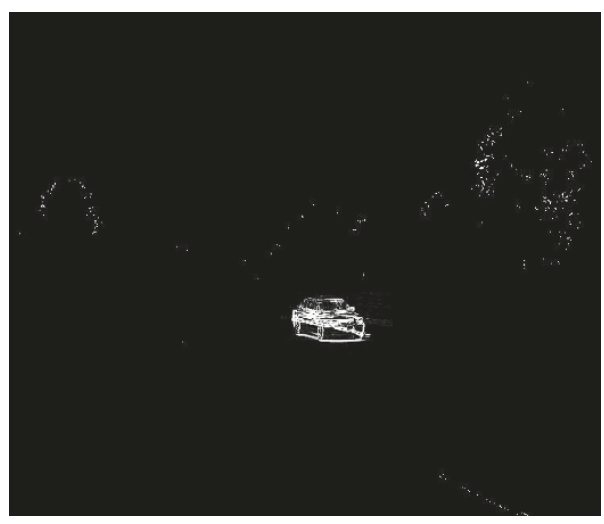

(c)

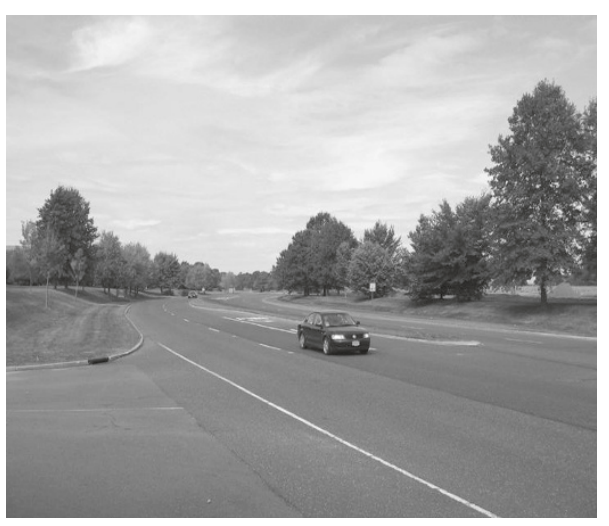

(b)

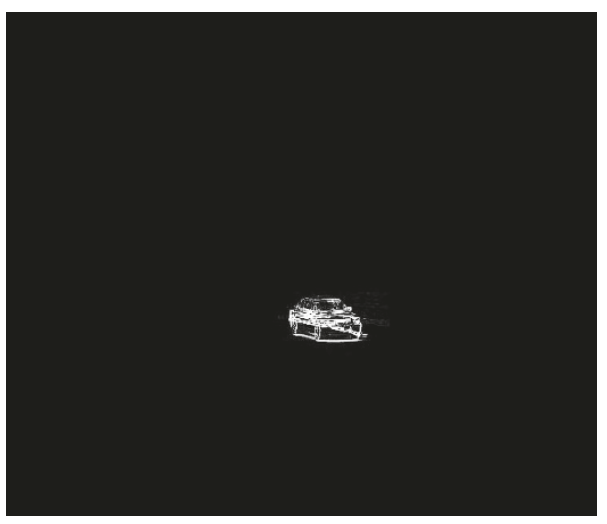

(d)

Figure 6: Result of background subtraction.

matching, we obtain best one-to-one pair match of the two sets of feature points. Following the shape context registration process, we obtain transform parameters $a$ from (5). A warped image is generated by transforming Figure 5(a). The difference image of the warped image and the reference image is shown in Figure 5(c). The maximal overlap is identified and the coding cost is reduced. Then we only transmit the original image and the difference image together with the warping transform parameters to the monitoring centering to reduce energy on transmission.

\subsection{Background subtraction to exploit temporal correlation}

The top two images in Figure 6 are taken by the same sensor. Figure $6(\mathrm{a})$ is the background that is to be transmitted to the monitoring center. When a new image is captured in the same sensor, the background subtraction algorithm described in Section 2.2 is employed for target detection. Figure 6(b) is captured with a target, a car, on it. Figure 6(c) is the result of the background subtraction. The car is successfully detected. At the same time, some small areas of the tree movement are also detected. Those areas can be viewed as noise and will be eliminated by a size filter. The detected areas with small size are considered as noises. Figure 6(d) shows the result after applying size filter. Only the car is left on this image. The sensor then transmits only a rectangle block containing this target area to the monitoring center.

\subsection{Energy saving in collaborative image transmission}

The energy saving on background subtraction is dependent on the size of the targets and how often the targets are detected. The energy saving on the image matching is dependent on the ratio of overlaps. In this experiment, we consider the case that each sensor transmits its background and the detected target area shown in Figure 6 to the monitoring center. In this case, the target is in the area of $48 \times 36$, which is $1 / 64$ of the entire image. When calculating the total energy consumption in additional image processing introduced in this collaborative image transmission, we adopt the unit energy consumptions of an $m$-bits addition and multiplication operation as $E_{\text {add }}=3.3 \times 10^{-5} \mathrm{~m} \mathrm{~mW} / \mathrm{MHz}$ and $E_{\text {mult }}=3.7 \times 10^{-5} \mathrm{~m}^{3} \mathrm{~mW} / \mathrm{MHz}$, assuming that SA 1110 works on $206 \mathrm{MHz}$. Table 1 shows the energy consumption on transmission, processing on image registration, as well as the additional processing in background subtraction. The 
TABLE 1: Energy consumption comparison with and without the proposed collaborative image transmission scheme.

\begin{tabular}{lcc}
\hline & W collaboration & W/O collaboration \\
\hline $\begin{array}{l}\text { Energy on image } \\
\text { transmission }(\mathrm{J})\end{array}$ & 0.577 & 1.7 \\
$\begin{array}{l}\text { Additional energy for } \\
\text { image registration }\end{array}$ & 0.222 & - \\
$\begin{array}{l}\text { Additional energy for } \\
\text { background subtraction }\end{array}$ & 0.006 & - \\
$\begin{array}{l}\text { Total energy consumption } \\
\text { Energy saving }\end{array}$ & 0.805 & 1.7 \\
\hline
\end{tabular}

saving in transmission energy due to reduced data transmission is about $1.1 \mathrm{~J}$, while the additional energy consumption due to the increase in collaborative processing is about $0.23 \mathrm{~J}$. Taking both types of energy consumption into consideration, we find that the total energy can be saved $53 \%$ by the proposed collaborative image transmission scheme.

\section{SUMMARY AND DISCUSSION}

In this paper, we described a novel collaborative image transmission scheme for wireless sensor networks. In our application, we consider exploiting both spatial and temporal correlations to save overall energy consumption on data transmission and processing. To exploit the spatial correlation between images in neighboring imaging sensors, after one image is transmitted to its neighboring imaging sensor along the route, we employ the image matching method involving image feature points to roughly register images in order to find out the maximal overlap. Then, we warp the original image and code the original image and the difference between the reference and the warped image. This will significantly reduce transmission energy comparing with transmitting two individual images independently. To exploit the temporal correlation between images in each sensor, we employ background subtraction algorithm on gradient image to detect target. We only transmit background image from each sensor to the monitoring center once. Whenever one or more targets are detected, only the regions of targets and their spatial locations are transmitted to the monitoring center. At the monitoring center, the whole image can be reconstructed by fusing the background and the target image as well as its spatial location. Experimental results show that the transmission energy can be greatly reduced. For the example we presented in this paper, the total energy, including both processing energy and transmission energy, has been saved 53\%.

This is the first attempt to apply collaborative signal processing principles to imaging sensor networks. The vast amount of image data these sensors collect and the intrinsic characteristics of these images pose significant challenge in how to efficiently compress and transport the sensor data wirelessly via multi-hop routing to a monitoring center with an acceptable quality-of-service guarantee. Because such a sensor network is usually severely constrained by battery power and processing capability, there are potential trade-offs that need to be carefully studied so that the overall system energy consumption can be minimized while still maintaining an acceptable image quality, for instant, recognition and decision at the remote monitoring center to carry out its surveillance tasks.

We would like to point out that the proposed scheme is designed for the application that neighbouring sensor images have high correspondance. Current typical scenario for such application can often be found in the outdoor environment. Therefore, we considered lighting changes in the outdoor environment during the day and proposed periodic update of background reference images. When the proposed algorithm is applied to indoor applications, additional attention on perspective distortion is needed to ensure that the correlation of the background remains sufficiently high to adopt the proposed scheme. With the increase of the processing power of sensor nodes, we will develop more complex algorithm to deal with the complicated cases in which the imaged scene presents foreground and background objects so as to avoid the possibility to compensate one image with respect to another with the transformation defined in (7).

\section{ACKNOWLEDGMENT}

This research is supported by FIT Allen Henry Endowment Fund.

\section{REFERENCES}

[1] D. Estrin, L. Girod, G. Pottie, and M. Srivastava, "Instrumenting the world with wireless sensor networks," in Proceedings of IEEE International Conference on Acoustics, Speech and Signal Processing (ICASSP '01), vol. 4, pp. 2033-2036, Salt Lake City, Utah, USA, May 2001.

[2] C. Savarese, J. M. Rabaey, and J. Beutel, "Locationing in distributed ad-hoc wireless sensor networks," in Proceedings of IEEE International Conference on Acoustics, Speech and Signal Processing (ICASSP '01), vol. 4, pp. 2037-2040, Salt Lake City, Utah, USA, May 2001.

[3] S. S. Pradhan, J. Kusuma, and K. Ramchandran, "Distributed compression in a dense microsensor network," IEEE Signal Processing Magazine, vol. 19, no. 2, pp. 51-60, 2002.

[4] D. Li, K. D. Wong, Y. H. Hu, and A. M. Sayeed, "Detection, classification, and tracking of targets," IEEE Signal Processing Magazine, vol. 19, no. 2, pp. 17-29, 2002.

[5] Y. J. Zhao, R. Govindan, and D. Estrin, "Residual energy scan for monitoring sensor networks," in Proceedings of IEEE Wireless Communications and Networking Conference (WCNC '02), vol. 1, pp. 356-362, Orlando, Fla, USA, March 2002.

[6] R. Wagner, R. Nowak, and R. Baraniuk, "Distributed image compression for sensor networks using correspondence analysis and super-resolution," in Proceedings of IEEE International Conference on Image Processing (ICIP '03), vol. 1, pp. 597-600, Barcelona, Spain, September 2003.

[7] A. D. Liveris, Z. Xiong, and C. N. Georghiades, "A distributed source coding technique for correlated images using turbocodes," IEEE Communications Letters, vol. 6, no. 9, pp. 379381, 2002. 
[8] B. Girod, A. Aaron, S. Rane, and D. Rebollo-Monedero, "Distributed video coding," Proceedings of the IEEE, vol. 93, no. 1, pp. 71-83, 2005, IEEE special issues on advances in video coding and delivery, 2004.

[9] X. Zhu, A. Aaron, and B. Girod, "Distributed compression for large camera arrays," in Proceedings of IEEE Workshop on Statistical Signal Processing (SSP '03), pp. 30-33, St. Louis, Mo, USA, September-October 2003.

[10] R. Puri and K. Ramchandran, "PRISM: a video coding architecture based on distributed compression principles," Tech. Rep. UCB/ERL M03/6, EECS Department, University of California, Berkeley, Calif, USA, 2003, http://www.eecs. berkeley.edu/ kannanr/PRISM/.

[11] K. Akkaya and M. Younis, "A survey on routing protocols for wireless sensor networks," Ad Hoc Networks, vol. 3, no. 3, pp. 325-349, 2005.

[12] S. Belongie, J. Malik, and J. Puzicha, "Shape matching and object recognition using shape contexts," IEEE Transactions on Pattern Analysis and Machine Intelligence, vol. 24, no. 4, pp. 509-522, 2002.

[13] R. Bhargava, H. Kargupta, and M. Powers, "Energy consumption in data analysis for on-board and distributed applications," in Proceedings of the ICML Workshop on Machine Learning Technologies for Autonomous Space Applications, Washington, DC, USA, August 2003.

[14] K. Akkaya and M. Younis, "An energy-aware QoS routing protocol for wireless sensor networks," in Proceedings of the 23rd International Conference on Distributed Computing Systems Workshops (ICDCSW '03), pp. 710-715, Providence, RI, USA, May 2003.

[15] A. Mainwaring, J. Polastre, R. Szewczyk, D. Culler, and J. Anderson, "Wireless sensor networks for habitat monitoring," in Proceedings of the ACM International Workshop on Wireless Sensor Networks and Applications (WSNA '02), pp. 88-97, Atlanta, Ga, USA, September 2002.

[16] W. Ye, J. Heidemann, and D. Estrin, "An energy-efficient MAC protocol for wireless sensor networks," in Proceedings of the 21st Annual Joint Conference of the IEEE Computer and Communications Societies (INFOCOM '02), vol. 3, pp. 1567-1576, New York, NY, USA, June 2002.

[17] O. Javed, K. Shafique, and M. Shah, "A hierarchical approach to robust background subtraction using color and gradient information," in Proceedings of IEEE Workshop on Motion and Video Computing (MOTION '02), pp. 22-28, Orlando, Fla, USA, December 2002.

[18] A. Mittal and D. Huttenlocher, "Scene modeling for wide area surveillance and image synthesis," in Proceedings of IEEE Conference on Computer Vision and Pattern Recognition (CVPR '00), vol. 2, pp. 160-167, Hilton Head Island, SC, USA, June 2000.

[19] A. Monnet, A. Mittal, N. Paragios, and V. Ramesh, "Background modeling and subtraction of dynamic scenes," in Proceedings of IEEE Conference on Computer Vision and Pattern Recognition (CVPR '00), Hilton Head Island, SC, USA, June 2000.

[20] Y. Ivanov, A. Bobick, and J. Liu, "Fast lighting independent background subtraction," International Journal of Computer Vision, vol. 37, no. 2, pp. 199-207, 2000.
Min Wu received his B.S. degree from Tsinghua University in 1993, M.S. degree from University of Science and Technology of China, in 1997, and Ph.D. degree from Department of Electrical and Computer Engineering, University of Missouri-Columbia in 2005. He served as Lecturer with the Department of Automation, University of Science and Technology of China, from 1997 to 2000. In 2005, he joined MAKO Surgical Corp. at Fort Lauderdale, FL, as senior software engineer. His current research interests are focused on biomedical image processing, wireless image/video transmission, and wireless sensor network. $\mathrm{He}$ is a member of Sigma $\mathrm{Xi}$ and IEEE, and was named one of the three finalists for 2003 Association for the Advancement of Medical Instrumentation (AAMI) Young Investigator Competition.

Chang Wen Chen received the B.S. degree from University of Science and Technology of China in 1983, M.S.E.E. degree from University of Southern California, Los Angeles, in 1986, and Ph.D. degree from University of Illinois at Urbana-Champaign, in 1992. He has been Allen S. Henry Distinguished Professor in the Department of Electrical and Computer Engineering at the Florida Institute of Technology since July 2003. Pre-

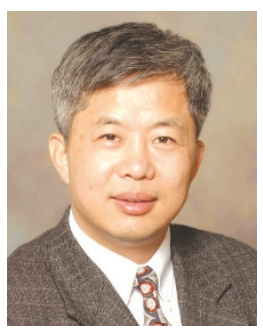
viously, he was on the faculty at the University of MissouriColumbia and at the University of Rochester. From 2000 to 2002, he served as the Head of Interactive Media Group at the David Sarnoff Research Laboratories in Princeton, NJ. He has received a number of awards including the Sigma Xi Excellence in Graduate Research Mentoring Award in 2003. He was elected an IEEE Fellow in 2004. He has been the Editor-in-Chief for IEEE trans. Circuits and Systems for Video Technology (T-CSVT) since January 2006. He has been an Editor for a number of journals, including Proceedings of IEEE, IEEE trans. Multimedia, IEEE T-CSVT, IEEE Multimedia, Journal of Visual Communication and Image Representation. He served as the the Chair of the Technical Program Committee for ICME 2006 held in Toronto, Canada in July 2006. 